\title{
MedienPädagogik
}

Zeitschrift für Theorie und Praxis der Medienbildung

\section{Wer versetzt wen oder was wohin - und wozu?}

\section{Eine kritische Auseinandersetzung mit Augmented und Virtual Reality in schulischen Bildungsangeboten}

\author{
Nicola Przybylka $\bullet$ \\ ${ }^{1}$ Ruhr-Universität Bochum
}

\section{Zusammenfassung}

Augmented Reality (AR) und Virtual Reality (VR) werden verstärkt in schulischen Anwendungskontexten diskutiert. Es wird erwartet, dass beide Technologien neue Möglichkeiten für das Lehren und Lernen eröffnen: Schülerinnen und Schüler sollen zum einen in vergangene Zeiten oder an unerreichbare Orte versetzt werden und diese zuweilen aus der Perspektive bzw. im Körper einer anderen Person erleben. Zum anderen wird das Potenzial gesehen, Objekte oder Personen in das Klassenzimmer zu holen und zu untersuchen bzw. zu befragen. Der Beitrag geht diesem Versetzen in andere Zeiten, Räume und Körper nach, das mit Augmented und Virtual Reality verknüpft wird, und stellt dabei eine Eigenschaft in den Fokus, die von der Autorin als 〈Entrahmung der Bildlichkeit) bezeichnet wird. Dieses Spezifikum wird jedoch nicht als der Technik per se eingeschrieben betrachtet. Stattdessen schlüsselt der Beitrag dessen Entstehung als Zusammenspiel zwischen der Funktionslogik beider Technologien und den diskursiven Zuschreibungen (an den Lernerfolg) auf. Anhand von AR- und VR-Anwendungen für die Fächer Sport, Geschichte und Geografie wird beispielhaft diskutiert, wie 
die (Entrahmung der Bildlichkeit) thematisch ausgestaltet wird und wie damit einhergehende Wissens- und Machteinschreibungen neue didaktische Herausforderungen schaffen.

\title{
Who Transports Whom or What Where to - and What for? A Critical Approach to Augmented and Virtual Reality in Educational Contexts
}

\begin{abstract}
Augmented Reality (AR) und Virtual Reality (VR) have been increasingly discussed in the context of their application at schools. AR and VR are expected to offer new possibilities for studying and teaching: on the one hand, students are supposed to be transported to past times or unreachable places and should experience those from the perspective or in the body of another person. On the other hand, there is the potential to bring objects or people into the classroom in order to investigate or interview them. This article analyzes the aforementioned transportation into other times, places and bodies, which is connected with AR and VR. Therefore, it focusses on one feature which the author calls «picture without frames. This characteristic of AR and VR is not regarded as a fixed element or as a part of the technology per se. Instead of that it is explained as the interplay of the operational logic of both technologies and the discourse (about the learning success). With the help of Augmented and Virtual Reality applications for the subjects Sports, History and Geography it will be illustrated how the spicture without frames influences the content of these applications. Moreover, it will be discussed, how associations of knowledge and power offer new challenges for didactics.
\end{abstract}




\section{AR und VR: (Schon) ein Thema für die Schule? ${ }^{1}$}

Augmented Reality (AR) und Virtual Reality (VR) haben - nach einem Hype in den 1990ern und einer anschliessenden Phase der Ernüchterung über den Gehalt der Technologien in den 200oern ${ }^{2}$ - in den letzten Jahren erneut an ökonomischer und gesellschaftlicher Relevanz gewonnen. Beide Technologien werden mittlerweile in industriellen und unternehmerischen Kontexten, unter anderem in der Marktforschung und Produktwerbung, ${ }^{3}$ im Architektur- und Bauwesen, im medizinischen Sektor oder auch in der Automobil- und Tourismusbranche eingesetzt. Durch immer kostengünstigere VR-Technik und die flächendeckende Verbreitung digitaler Endgeräte wie Smartphones und Tablets, von denen relativ neue Modelle sowohl AR-Inhalte wiedergeben als auch $360^{\circ}$-Videos abspielen können, haben Augmented und Virtual Reality zudem Einzug in die Lebenswelten vor allem junger Menschen erhalten. Das Mobile-Game Pokémon Go von 2016, AR-Filter zur Bearbeitung von Fotos oder Videos, die Erweiterung von Produktlinien bekannter Spielzeughersteller ${ }^{4}$ oder die zunehmende Popularität von VR-Games sind nur einige Beispiele für die vermehrte Nutzung beider Technologien. Nach Ausbruch der Coronapandemie im Jahr 2020 haben darüber hinaus global führende Technologiekonzerne wie Microsoft und Facebook bzw. Meta ihr Portfolio stärker um AR- und VR-Produkte erweitert. So startete Microsoft zu Beginn des Jahres 2021 die AR/VRPlattform Mesh der damals noch unter dem Namen Facebook agierende Konzern Meta richtete im Herbst 2020 mit Facebook Reality Labs (nach der

1 Der Aufsatz entstand im Rahmen des Projekts «Digitalisierung in der Ausbildung von Lehramtsstudierenden: Orientierung und Gestaltung ermöglichen» an der Ruhr-Universität Bochum, das von der gemeinsamen «Qualitätsoffensive Lehrerbildung» von Bund und Ländern mit Mitteln des Bundesministeriums für Bildung und Forschung unter dem Förderkennzeichen 01JA2040 gefördert wird. Die Verantwortung für den Inhalt dieser Veröffentlichung liegt bei der Autorin.

2 Im Vergleich zu VR zeichnete sich diese Entwicklung bei AR ein paar Jahre später ab.

3 So kann mit AR ein gewünschtes Produkt vor dem Kauf probeweise im Raum platziert oder am Körper 〈getragen〉 werden. Ein Beispiel hierfür ist die ARApp des Möbelherstellers IKEA oder des Augenoptik-Unternehmens Apollo.

4 Die App Applaydu augmentiert mit einem QR-Code das Spielzeug aus einem Überraschungs-Ei. Auch Lego nutzt mit der Produktlinie Hidden Side ARTechnologie, um seine analogen Angebote mit computergenerierten Visualisierungen zu erweitern. 
Umbenennung des Konzerns jetzt nur noch Reality Labs) einen eigenen Forschungsbereich zu Augmented und Virtual Reality ein. Bei der Veranstaltung Facebook Connect wurde nicht nur ebendieser Forschungsbereich vorgestellt, sondern mit AR- und VR-Technologie auch eine technische Lösung gegen die coronabedingte räumliche und damit physische Distanz präsentiert:

«What we're missing is this feeling of presence, the feeling of actually being right there with someone else with all of the different sensations that that includes. So that's what the whole fields of virtual and augmented reality are about: delivering that sense of presence» (Facebook Connect | Keynote 2020).

Der unterrichtliche (Förder-)Bedarf an digitalen Lehr- und Lernangeboten wurde spätestens mit dem im Jahr 2019 verabschiedeten DigitalPakt Schule politisch anerkannt und erlangte mit der coronabedingten Schliessung der Schulen und Universitäten eine neue Dringlichkeit. Zwar gelten Augmented und Virtual Reality Medienangebote noch nicht als etablierte didaktische Lehr- und Lernobjekte, schulische Anwendungskontexte werden jedoch verstärkt diskutiert (Radu 2014; Liu u. a. 2017; Maas und Hughes 2020; Jauch 2019). Beide Technologien sind zudem seit einigen Jahren auf der Bildungsmesse Didacta vertreten, der Ernst Klett Verlag bietet eine ARApp für den Fremdsprachenunterricht an und der Lehrerclub der Stiftung Lesen entwickelt in Kooperation mit der Google Zukunftswerkstatt Unterrichtskonzepte für den Einsatz von AR und VR in der Schule. Darüber hinaus informieren Medienzentren verschiedener Städte und Bundesländer auf ihren Internetpräsenzen oder in kollaborativ erstellten Sammlungen über AR- und VR-Apps für diverse Unterrichtsfächer (kmz 2020; Wössner o. J.; Medienzentrum Pforzheim-Enzkreis o. J.). 


\section{Ziel des Beitrags}

«Wenn sich Diskurse und Ideologien in Materialien einzuschreiben vermögen, Dinge Geschichten erzählen und Artefakte zum Auslöser von Handlungen werden, verdienen Medien und Materialitäten unsere wissenschaftliche Aufmerksamkeit. [...] Indem Gegenstände durch eine bestimmte Begrifflichkeit kontextualisiert und im Sinne einer von uns selbst vorgenommenen Autopsie erst eine wissenschaftliche Evidenz zugesprochen bekommen, werden sie auch erst von uns zum Sprechen gebracht.» (GfM, Gesellschaft für Medienwissenschaft 2019)

In diesem Sinne werden im Folgenden AR- und VR-Angebote in den Blick genommen, die entweder speziell für die Schule oder allgemein für die Vermittlung von fachspezifischem Wissen konzipiert wurden. Die Analyse fokussiert dabei jene Potenziale von Augmented und Virtual Reality, die den Technologien in bildungs- und lerntheoretischen Auseinandersetzungen sowie vonseiten der Produzierenden für den Lerngehalt und -erfolg zugeschrieben werden. Diese Eigenschaften werden jedoch nicht als den Technologien fest eingeschriebene Spezifika verstanden. Stattdessen versucht der Beitrag, deren Entstehung als Wechselspiel zweier Faktoren aufzuschlüsseln. Hierzu veranschaulicht zunächst ein historischer Umriss die grundsätzliche technische Operationsweise von AR und VR. Darauf aufbauend beschreibt der Beitrag, wie diese Medialität der Technologien semantisch im Hinblick auf Bildungskontexte ausgestaltet wird. Dazu werden sowohl die oben erwähnten Empfehlungen von Landesmedienzentren als auch mediendidaktische und lerntheoretische Auseinandersetzungen mit AR und VR aus dem deutschsprachigen Raum untersucht. Zusätzlich berücksichtigt die Analyse die Internetauftritte und Leitbilder jener Akteurinnen und Akteure, die die Anwendungen für den Bildungskontext entwickeln (öffentlich-rechtliche Sendeanstalten und nicht originär im Bildungssektor angesiedelte Unternehmen) oder die technische Infrastruktur bereitstellen, u. a. Grosskonzerne wie Facebook/Meta oder Google. Anschliessend werden Inhalte von AR- und VR-Anwendungen für 
die Fächer Geschichte, Geografie und Sport ${ }^{5}$ vorgestellt und in Beziehung zu den technischen Möglichkeitsbedingungen und diskursiv hervorgebrachten Narrativen gesetzt. Den sich aus Diskurs und Technologie ergebenden Wissens- und Machteinschreibungen ${ }^{6}$ werden anschliessend zwei Problemaufrisse gegenübergestellt, die über die genannten Fächer hinaus für AR- und VR-Angebote im Bildungskontext allgemein diskutiert werden können.

Es sei angemerkt, dass nicht von einer durch das Medium vordefinierten Nutzungsweise ausgegangen oder ein direkter Rückschluss auf die Rezeptionserfahrung gezogen wird.

«Weder passt sich die Person allein an den vordefinierten Gebrauch von Medien an, noch unterliegen die medialen Gebrauchsformen einer determinierten oder determinierenden Intentionalität und Funktionalität durch die Teilnehmer*innen» (Stern 2019, 49).

Die Operationsformen der Technologien selbst und deren narrative Ausdeutung über einen wie auch immer gestalteten Mehrwert für das Lernen legen jedoch Bedingungen offen, die bestimmte Nutzungsweisen und Wahrnehmungsformen ermöglichen bzw. verhindern.

5 Die Auswahl der Fächer liegt zum einen in dem Projekt begründet, in dem die Autorin als wissenschaftliche Mitarbeiterin beschäftigt ist und das sich mit AR und VR in Bildungskontexten auseinandersetzt. Zum anderen bietet sich die Fächerkombination an, um die für die Analyse relevanten Kategorien Zeit, Raum und Körper adressieren zu können.

6 Macht soll im Verständnis von Michel Foucault und seiner Konzeption zur Gouvernementalität nicht als repressive, negative Instanz aufgefasst werden. Stattdessen schafft sie bestimmte Möglichkeitsräume, die etwas als denkund sagbar, sichtbar und machbar ausweisen und damit neue Gegenstände, Wissensformen und Subjektivitäten hervorbringen (Dreesen, Kumięga, und Spieß 2012). 


\section{Augmented und Virtual Reality - Bild ohne Rahmung?}

Augmented Reality zeichnet sich dadurch aus, dass mittels eines digitalen Endgeräts computergenerierte, überwiegend visuelle Informationen wie Texte oder dreidimensionale Objekte in das sichtbare Umfeld des Rezipierenden eingeblendet werden. ${ }^{7}$ Diese Überlagerung von digitaler Information und wahrgenommenem Raum erfolgt in sogenannter Echtzeit und ist kontextsensitiv bzw. positionsgebunden (Azuma u. a. 2001; Buchner 2017). Ideengeschichtlich reicht AR bis in die 1960er-Jahre zum Electrocular zurück, das der Unterstützung von mechanischen Instandhaltungsprozessen und der Navigation beim Flugverkehr dienen sollte. Der Name〈Augmented Reality> etablierte sich jedoch erst in den 1990er-Jahren. Während ursprünglich zur Rezeption von Augmented Reality technisch ein HeadMounted-Display (HMD) als Standard galt, hat sich für die alltägliche Nutzung mittlerweile ein digitales Endgerät mit integrierter Kamera (Smartphone oder Tablet) durchgesetzt (Lenzen 2020).

Als Virtual Reality werden im Folgenden sowohl digitalisierte $360^{\circ}$-Aufnahmen ${ }^{8}$ als auch vollständig computergenerierte Bildräume bezeichnet, die mithilfe bestimmter Hardware-Komponenten, zum Beispiel mit einer VR-Brille oder einem Head-Mounted-Display, rezipiert werden. Neben visuellem und auditivem Feedback können auch taktile Reize über Controller ausgegeben werden. Häufig ist ein formalästhetischer Realitätsgrad, die Möglichkeit der Interaktion mit Objekten innerhalb der Virtual Reality sowie eine damit in Zusammenhang gebrachte subjektive Präsenz-Erfahrung Teil von VR-Definitionen (Milgram und Kishino 1994; Buehler und Kohne 2020; Zobel u. a. 2018). Die Ursprünge des Strebens nach einer möglichst auf Realitätsnähe ausgerichteten und die Betrachter

7 Azuma et al. weisen darauf hin, dass die digitalen Informationen auch so gestaltet sein können, dass Objekte im sichtbaren Raum derart überlagert werden, dass diese durch Augmented Reality aus dem Blickfeld der Rezipierenden verschwinden: «Certain AR applications also require removing real objects from the perceived environment, in addition to adding virtual objects» (Azuma u. a. 2001, 34).

$8360^{\circ}$-Aufnahmen sind nicht computergeneriert, sondern werden mit einer $360^{\circ}$-Kamera mit mindestens zwei Kameralsinsen erstellt, die auch als omnidirektionale Kamera oder Vollsphärenkamera bezeichnet wird. Eine sogenannte Stitching-Software stellt die digitalisierten Bilder der Kameralinsen anschließend zu einem kugelförmigen $360^{\circ}$-Bild zusammen (Neußl 2018, 42). 
umhüllenden Bildästhetik können zeitlich unterschiedlich angesetzt werden. Die Panoramamalerei im 18. Jahrhundert, die Stereoskopie Mitte des 19. Jahrhunderts oder das 1962 vom Kameramann Morton Heilig patentierte Sensorama - eine kabinenartige Apparatur, die multisensorische Filmerlebnisse unter didaktischen Leitlinien ermöglichen sollte (Schröter 2004, 186) - können als unterschiedliche ideengeschichtliche Vorläufer der Virtuellen Realität angesehen werden (Henze u. a. 2019; Grau 2001).

Als Zäsur in der technologischen Entwicklung sowohl von Virtual als auch Augmented Reality gilt jedoch Ivan Sutherlands Sword of Damocles von 1968. Die Assoziation mit dem Damoklesschwert wurde durch die bedrohlich über dem Kopf der Rezipierenden angebrachte brillenartige Konstruktion hervorgerufen (Abb. 1).

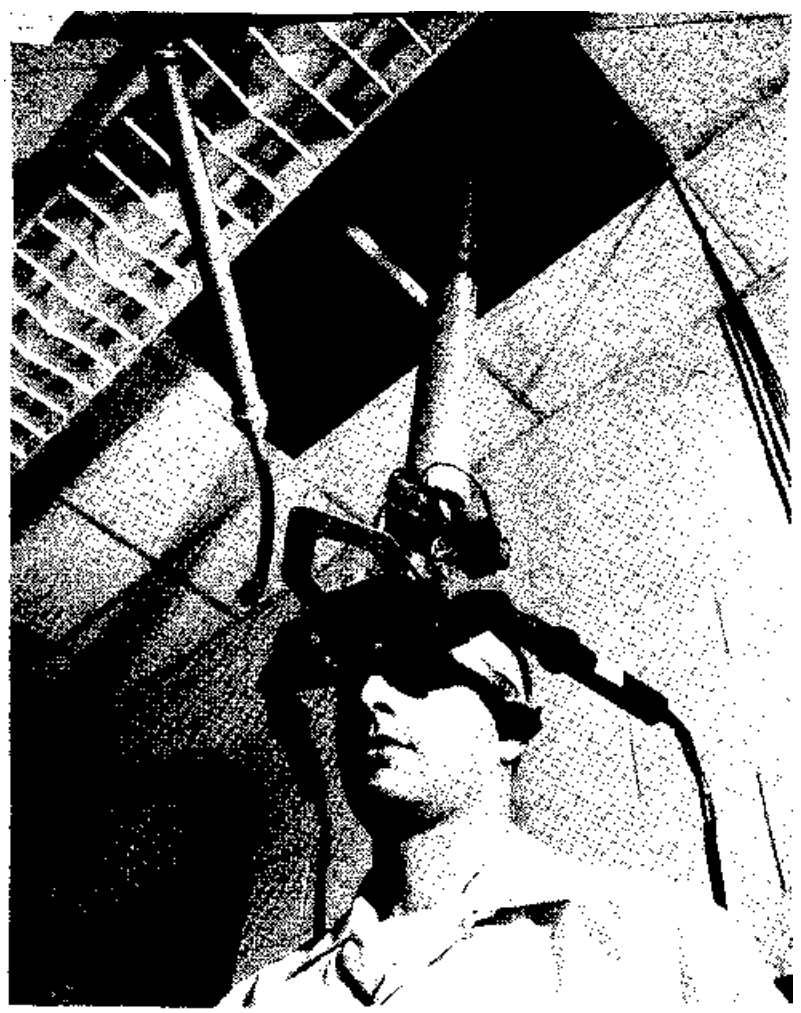

FIGURE 4-The ultrasonic bead position sensor in use

Abb. 1: Das Sword of Damocles (Sutherland 1968, 760). 
Die gemeinhin als erstes HMD angesehene Vorrichtung konnte noch keine umfassende computergenerierte Umgebung darstellen und berechnen. Stattdessen wurden durch als Display verwendete Brillengläser gitterartige Formen in das Sichtfeld projiziert. Somit handelt es sich eher um eine Anreicherung des sichtbaren Raumes im Sinne von Augmented Reality (Kasprowicz 2019, 299). Mit dem Sword of Damocles wurde jedoch eine technische Innovation für die Bildrezeption etabliert, die wegweisend für die nachfolgende Entwicklung beider Technologien war: Der Informatiker und Computergrafikpionier Sutherland vereinte in dem HMD das binokulare (beidäugige) Bild der Stereoskopie ${ }^{9}$ mit dem Rundumblick der Panoramamalerei. Entscheidend war, dass Sutherland mit seinem Programm das Entpacken und Verarbeiten von Daten in Echtzeit realisierte und damit die Synchronisierung der dargestellten Objekte mit der Kopfbewegung und Blickrichtung des Trägers bzw. der Trägerin des HMDs ermöglichte. Im Unterschied zum Panorama, das auch über den fokussierten Bildausschnitt hinaus existiert, aktualisierte sich das Bild im Display je nach Blickposition - der dreidimensionale Bildraum wurde damit virtuell (Schröter 2004, 191). In dem 1968 veröffentlichten Aufsatz A head-mounted three dimensional display beschreibt Sutherland diese technologische Neuerung folgendermassen:

«The fundamental idea behind the three-dimensional display is to present the user with a perspective image which changes as he moves. [...] The image presented by the three-dimensional display must change in exactly the way that the image of a real object would change for similar motions of the user's head.» (Sutherland 1968, 757)

Daraus abgeleitet etablierte sich mit Augmented und vor allem Virtual Reality ein neuer Bildstatus, der noch heute medienkomparativ von anderen Bewegtbildmedien abgegrenzt wird. «Mit der VR-Brille kann man sich in alle Richtungen umsehen. Ganz anders als beim Fernsehen» («Virtual Reality - Schöne neue Welt?» 2020).

9 Dabei wird für jedes Auge ein eigenes Bild über eine separate Linse präsentiert. Durch die optische Überlagerung der beiden Bilder entsteht so der Eindruck eines dreidimensionalen Bildes. 
In Beschreibungen dieser spezifischen Bildlichkeit von AR und VR drückt sich die Erwartung - oder vielmehr die Utopie - aus, mithilfe dieser Technologien den Bildrahmen, der den Blick determiniere und einschränke, zu überwinden. Der Bildrahmen bezieht sich nicht nur auf das gemalte Bild, sondern wird auch als Metapher beim Film verwendet. Vermutlich wurde sie durch Rudolf Arnheim prominent, der sich bereits 1932 in Film als Kunst mit der Notwendigkeit einer Rahmung der Wirklichkeit beim Film durch eine bestimmte Wahl der Perspektive, Grösseneinstellung, Brennweite etc. auseinandersetzte. Das Filmbild grenzt sich damit vom 〈randlosen〉 Sehen des menschlichen Auges ab (Arnheim 1932). ${ }^{10}$ Beim televisuellen Bild wird diese Rahmung analog auch als «Fenster» betitelt (Hicketier 1999). Der Bildrahmen ist dabei mehr als eine visuelle Eingrenzung; er positioniert gleichzeitig das betrachtende Subjekt auf eine bestimmte Art und Weise, prägt dessen Wahrnehmung und Deutung des Gezeigten. Der Wegfall dieser als fremdbestimmt empfundenen Grenzen durch eine Verräumlichung des Bildes wird umgekehrt mit einer souveränen Kontrolle über eben diesen Bildraum und mit einer Freiheit des Blicks verknüpft. Die Wahrnehmung sei damit wieder auf die Physiologie des eigenen Auges zurückgeworfen. Ein nicht sichtbares Feld der dargestellten Welt - filmtheoretisch unter dem Begriff hors-champ gefasst - existiere bei AR und VR damit nicht mehr. Die passive Rolle des Betrachtenden wandelt sich in einen aktiven Beobachterstatus:

«Die Frage nach dem, was uns die Kadrierung [der festgelegte Rahmen des Bildausschnitts] verwehrt und was im Off sein könnte, lässt sich nun beim Betrachten von $360^{\circ}$ Videos mit einer einfachen Kopfbewegung beantworten. [...] Dadurch wird die Kadrierung aufgehoben, da das Bild an sich keine Grenzen mehr aufweist» (Neußl 2018, 80).

Chris Milk, der durch einen TED-Talk im Jahr 2015 das Label von VR als «empathy machine» massgeblich geprägt hat, fasst diese als Mehrwert ausgewiesene Eigenschaft folgendermassen zusammen:

10 Ich danke Felix Hasebrink für diesen Hinweis, der sich im Rahmen seiner Dissertation Making $O f(f)$. Die Rückseiten des Films mit dem hors-champ und horscadre beschäftigt. 
«I mean, all the media that we watch - television, cinema - they're these windows into these other worlds. [...] But I don't want you in the frame, I don't want you in the window, I want you through the window, I want you on the other side, in the world, inhabiting the world.» (Milk 2015)

Übertragen auf AR-Anwendungen, die über ein Tablet oder Smartphone rezipiert werden, wäre diese 〈Blickfreiheit〉 das Betrachten dreidimensionaler Objekte von jedem beliebigen Winkel aus. So kann das Gezeigte mit Fingergesten gedreht oder die Perspektive durch die eigene Bewegung im Raum verändert werden. ${ }^{11}$ Im Folgenden werden dieser Bildstatus und die damit verbundenen Zuschreibungen als 〈Entrahmung der Bildlichkeit〉 bezeichnet.

\section{Vom entrahmten Bild zur Immersion}

Das angenommene Wegfallen der visuellen Rahmung wird dabei mit dem Phänomen der Immersion assoziiert, meist als eine Art Eintauchen in einen medienvermittelten Inhalt umschrieben. Immersion wird sowohl in Form von subjektiven Erfahrungen validiert oder als Qualitätsmerkmal aufseiten der Apparatur angesiedelt. Obwohl ein immersives Rezeptionserlebnis verschiedenen Medien attestiert wurde und wird, hat der Begriff seit den 1990ern insbesondere im Kontext computergenerierter (Spiele-)Welten Konjunktur. Mittlerweile ist er auch fester Bestandteil von AR- und VR-Definitionen. Die Medienkulturwissenschaftlerin Robin Curtis stellt jedoch fest, dass trotz vielfacher Bezugnahmen die Bedeutung des Begriffs überwiegend unklar sei: «Despite this prevalence, (immersion〉 nevertheless remains a relatively unspecific placeholder in present-day discourse» (Curtis 2016, 41). Ohne im Detail auf das viel diskutierte Phänomen der Immersion einzugehen, soll an dieser Stelle die klare Positionierung der Immersion bei dem Einsatz von AR und VR im Bildungskontext festgehalten werden. Entgegen einem negativ behafteten mediendeterminierten Distanzverlust

11 Mit der Umstellung der AR-Technik auf ein digitales Endgerät wird im Vergleich zur Rezeption über ein HMD ein Rahmen wieder deutlich sichtbar. Dieser Widerspruch bleibt bei Umschreibungen von AR-Anwendungen seitens der Entwicklerinnen und Entwickler jedoch meist unberücksichtigt. 
positioniert man sowohl auf Unternehmendenseite (z. B. das Institute for Immersive Learning) als auch in wissenschaftlichen Auseinandersetzungen mit AR und VR in Lehr- und Lernarrangements Immersion zuweilen als Garant für den Lernerfolg: «Immersive Welten schaffen authentische Lernorte und helfen gleichzeitig, die technischen, kreativen und problemlösenden Fähigkeiten der Lernenden zu schärfen» (Mulders und Buchner 2020, 6) oder auch «Auf diese Weise bearbeitet der Lerner die Aufgabe nicht nur, er erlebt sie vielmehr immersiv, wodurch ein bestmögliches Lernergebnis garantiert wird» (Buehler und Kohne 2020, 90). Die Lernplattform unimersiv, die sich selbst als grössten Anbieter von VR-Trainings für die dominierenden VR-Hardwareanbieter beschreibt, koppelt Immersion bereits morphologisch an den Lernerfolg.

Die Funktionsweise bzw. die technologische Ermöglichungsbedingung des Entpackens von Bilddaten in Echtzeit verbindet augmentierte und virtuelle Bildlichkeit mit Utopien eines freiheitlichen Blicks, mit dem Aufbrechen des Bildrahmens oder dem immersiven Eintauchen in das Bild selbst. Bevor sich der Beitrag kritisch mit diesen Positionen auseinandersetzt, wird am Beispiel der Fächer Geografie, Geschichte und Sport aufgezeigt, wie im Schulkontext der technisch mögliche Darstellungsraum inhaltlich und konzeptionell ausgestaltet wird (Weich 2020,5) und wie die um AR und VR gespannten Narrative diese Gestaltung mitorganisieren. Dies führt auf den Titel des Beitrags zurück:

\section{Wer versetzt wen oder was wohin - und wozu?}

Bei der Vermarktung von AR-Anwendungen für den Bildungsbereich wird neben der Anreicherung des sogenannten Realraums auch mit dem Versprechen geworben, ein Objekt oder eine Person in den sichtbaren Raum des Rezipierenden hereinzuholen. Für das Fach Geschichte sieht Hauke Pölert dadurch

«eine Erweiterung des Unterrichts, die bis vor kurzem noch undenkbar war. Zeitzeugen kommen ins Klassenzimmer, Orte und Räumlichkeiten können auf verschiedenen Zeitebenen erkundet und historische Artefakte individuell untersucht werden.» (Pölert 2019) 
Mittels Echtzeit-Lokalisierung werden beispielsweise öffentliche Räume oder Plätze eines historischen Geschehens mit visuellen (und auditiven) Informationen angereichert. So präsentiert die App MauAR ehemalige Grenzverläufe der Berliner Mauer an den entsprechenden Orten der Hauptstadt. Im Geografieunterricht können über diese auf GPS-Daten basierenden AR-Formate gleichermassen ortsspezifische Informationen mitgeteilt werden. Mit ortsungebundenen AR-Formaten, die über bildliche Marker, QR-Codes oder Gesichtserkennung usw. operieren, wird für das Fach Geschichte der Anspruch erhoben, historische Quellen betrachten und untersuchen zu können (z. B. Civilisations AR). Mit der prominent für Schulen beworbenen App WDR AR 1933-45 soll darüber hinaus ein persönliches Gespräch mit Zeitzeuginnen und Zeitzeugen auch nach deren Ableben ermöglicht werden: «Indem die App die Zeitzeuginnen und Zeitzeugen als echte Hologramme in den persönlichen Raum der Nutzenden bringt, ermöglicht sie ein sehr nahes, authentisches Erleben der Menschen» (WDR 2019). Analog möchte die Software Google Expeditions für das Fach Geografie «Die Welt in Ihr Klassenzimmer holen» (Google, o. J.) und zum Beispiel einen Vulkan als 3D-Objekt von der Klasse betrachten lassen - «ganz so, als befände sich der Gegenstand tatsächlich im Raum» (Stiftung Lesen 2019, 3). ${ }^{12}$ Für das Unterrichtsfach Sport hingegen gibt es neben der Möglichkeit, die Anatomie des menschlichen Körpers zu visualisieren (Virtuali-Tee), bisher noch keine prominenten AR-Anwendungen für den schulischen Kontext.

Mit VR wird der Anspruch erhoben, etwas 〈erlebbar〉 zu machen, was jenseits des Erfahrungshorizontes der Schülerinnen und Schüler liegt. Dazu sollen sie in einen anderen Körper, eine andere Zeit oder einen anderen Raum hineinversetzt werden - an die anfänglichen Cyberspace-Utopien der 1990er-Jahre erinnernd heisst es dementsprechend: «In der virtuellen Realität können Sie überall alles sein» (Mittelstaedt 2021). Die überwiegende Kommunikationsabsicht von geschichtsvermittelnden Virtual Reality Formaten wird anhand Führerbunker VR deutlich. Die Virtual Reality Anwendung ist das erste Projekt der Reihe Time \& History des Hannoverschen Ingenieursbüros Nord XR, das eigentlich AR- und VR-Simulationen für die

$12 \mathrm{Ab}$ dem 30. Juni 2021 wird die App Google Expeditions eingestellt und deren Inhalte auf die Plattform Google Arts \& Culture migriert. 
Automobil- und Baubranche produziert: «Unsere Vision ist es, zerstörte oder nicht mehr zugängliche, historische Orte fotorealistisch in VR zu rekonstruieren, um es Menschen aus aller Welt zu ermöglichen, diese neu zu erleben» (Time \& History, o. J.). In Führerbunker VR sollen der Rezipient bzw. die Rezipientin in den Führerbunker im April 1945 hineinversetzt werden, dabei den passiven Beobachterstatus verlassen und «vom Zuschauer zum Anwesenden» werden, «der sich frei in der virtuellen Welt bewegen und jeden Winkel erforschen kann» (Time \& History, o. J.). Mit Virtual Reality möchte man so auf das Problem reagieren, Vergangenheit(en) in die Gegenwart zu übersetzen und diese nicht nur durch Archivierung von Quellen zugänglich, sondern «hautnah» (ZDF, o. J.) erlebbar machen: «Geschichte erleben, statt nur davon lesen» (Krech 2019). Für den Fachbereich Geografie ist beim Einsatz von Virtual Reality die Grundidee, nicht zugängliche oder weit entfernte Orte mit der Schulklasse bereisen zu können:

«Gerade das Fach Geographie bietet dank der Raumorientierung einen fruchtbaren Boden für Betrachtungen mit VR-Brillen. Wenn ein Gletscher, eine islamische Stadt oder das Mesozoikum nicht real erlebt werden können, ermöglicht VR (z. B. mit google earthVR oder google expeditions) Lernumgebungen mit intensiven Erlebnissen [...].» (Bürki und Buchner 2020, 49) ${ }^{13}$

Das 2018 erschienene Werbeheft zum Einsatz von Virtual Reality im Schulunterricht, das in Kooperation mit der Stiftung Lesen und der Google Zukunftswerkstatt entstanden ist, führt insbesondere Orte auf, die normalerweise nicht für Schulausflüge geeignet sind, wie das Flüchtlingslager Al-Zaatari in Jordanien oder das zerstörte Aleppo (Stiftung Lesen 2018, 7). Virtual Reality Anwendungen für das Fach Sport sind überwiegend im Gaming-Bereich angesiedelt und haben (noch) keinen expliziten unterrichtlichen Bezug. Die meisten Anwendungen für Sport und Bewegung sind an bekannte Sportarten angelehnt, deren Bewegungsabläufe entweder simuliert und damit erprobt, trainiert und optimiert werden können (z. B. Eleven Table Tennis) oder auch abgewandelt werden (Holopoint). Ferner gibt es eine wachsende Anzahl an Musik- und/oder Fitnessspielen wie Beat

13 Buchner und Bürki weisen jedoch explizit darauf hin, dass es bei VR-Exkursionen einer fachlichen Einordnung und didaktischen Begleitung bedarf; siehe Problemaufriss 1 in diesem Beitrag. 
Saber, Audioshield oder FitXR, zu deren Zweck bereits spezielle Brillen unter Gewichts- und Hygienekriterien (Vive Air) sowie eigene Fitnessgeräte (ICAROS) konzipiert werden. Insbesondere〈Extremsportarten〉, die die Vulnerabilität des eigenen Körpers vor Augen führen und Körpererfahrungen ermöglichen, die den allermeisten Menschen aufgrund der Begrenztheit der eigenen Physis verwehrt bleiben (z. B. Freiklettern in The Climb oder Boxen in Creed - Rise of Glory), scheinen im Sportkontext von Interesse (nextReality. Hamburg 2021).

Zusammenfassend wird durch die technologische Möglichkeit des Entpackens von Daten in Echtzeit und in Abstimmung mit der Blickrichtung und -perspektive diskursiv ein neuer Bildstatus verknüpft, der die Auflösung eines determinierenden Bildrahmens propagiert. In der inhaltlichen Ausgestaltung von AR- und VR-Anwendungen für den Bildungskontext schlägt sich diese 〈Entrahmung der Bildlichkeit〉 für die Fächer Geschichte, Geografie und Sport in einer beworbenen Zeit-, Orts und Körperungebundenheit thematisch nieder. Schülerinnen und Schüler sollen mit VR in historische Zeiten oder an unerreichbare Orte versetzt werden und diese zuweilen im Körper einer anderen Person erleben. Zum anderen wird bei AR von dem Potenzial gesprochen, Objekte oder Personen in das Klassenzimmer holen, untersuchen und befragen zu können. Die mit diesem Versetzen in andere Zeiten, Räume und Körper verknüpften Implikationen werden im Folgenden anhand zweier Aspekte kritisch diskutiert.

\section{Problemaufriss 1: Das (produktionsbezogene) Aussen}

Der durch AR und VR ermöglichte Rundumblick geht auf diskursiver Ebene meist mit einer wie auch immer gearteten Wahrhaftigkeit oder Authentizität der Lernsituation einher:

«Motiviert durch das Ziel, eine authentische mediale Umgebung zu schaffen, war der nächste logische Schritt in dieser Entwicklung der Versuch, den besagten Rahmen der Darstellung zu verlassen» (Stiftung Lesen 2018, 4). 
Dieses Narrativ verstärkt auch Chris Milk (s. o.): «So what I'm going to show you is not a view into the world, it's basically the whole world stretched into a rectangle» (Milk 2015). Vor diesem Hintergrund könnte der Titel der WDR-Dokumentation Inside Auschwitz - Das ehemalige Konzentrationslager in $360^{\circ}$ sowohl die Machart als $360^{\circ}$-Film beschreiben, als auch den Informationsgehalt der Dokumentation als «allumfassend» bewerben.

Die Annahme, dass der Bildraum nun keine Kadrierung mehr aufweise und lediglich auf die Physiologie des menschlichen Auges begrenzt sei, greift jedoch zu kurz. Wie bei allen technischen Medien gibt es auch bei AR und VR ein produktionsbezogenes Aussen (beim Film als hors-cadre bezeichnet), das sich in der apparativen Technik, der Hard- und Software, aber auch in den Daten, die in Echtzeit entpackt werden, materialisiert. Darüber hinaus zählen redaktionelle Prozesse, ästhetische und dramaturgische Entscheidungen etc. zu diesem Aussen. Wird die Entrahmung des Gezeigten und die daraus abgeleitete Blickfreiheit mit einer selbstbestimmten Wahl, einer neutralen und authentischen Allverfügbarkeit von Information gleichgesetzt, klammert diese Erzählung damit technische Ermöglichungsbedingungen, Implikationen, Deutungen und (Verwertungs-)Interessen aus, die ein produktionsbezogenes Aussen mit sich bringen. Somit sollte nicht nur der Inhalt der Anwendungen für Lehr- und Lernprozesse in Form eines sogenannten Faktenchecks reflektiert, sondern im medienbildenden Sinne auch thematisiert werden, wie das produktionsbezogene Aussen sowohl in Form des Mediums selbst als auch in Form von Produktionsprozessen und Autorenschaft Wahrnehmungsweisen und Wissensprozesse verändert.

Ein Kern des Faches Geschichte besteht darin, die Darstellung von Geschichte als Erzählung von den geschehenen Taten und Dingen zu erkennen und als eine mögliche, jedoch nicht als alleinige Perspektive einzuordnen. Virtual Reality stellt die Lehrkraft dabei verstärkt vor die Aufgabe, die Schülerinnen und Schüler auf den Konstruktionscharakter des Dargestellten und die Unmöglichkeit einer nachträglichen Zeitzeugenschaft hinzuweisen (Bunnenberg 2018; Wagner 2019; Jordan 2013). Auch die App WDR AR 1933-45, die mit ihrer AR-Anwendung den biografischen Erzählungen von Zeitzeuginnen und Zeitzeugen eine immerwährende Gültigkeit 
attestiert und damit eine Zeit-, Orts- und Kontextunabhängigkeit der Aussagen suggeriert, muss dahingehend problematisiert werden. Denn wie in anderen Medienformaten sind auch hier die biografischen Erinnerungen in eine Narration eingebunden und werden ästhetischen, technischen oder dramaturgischen Entscheidungen unterworfen. ${ }^{14}$ Der Einfluss der Interviewsituation und der eingesetzten Technik wird in den hauseigenen Making-ofs von WDR und ZDF zwar thematisiert - jedoch nicht, um das produktionsbezogene Aussen zu reflektieren, sondern um das technische Equipment auszustellen (Making of WDR History App, o. J.; «History 360 - das Making-of. Wie entstehen 360 Grad-Dokumentationen?» 2018). Gleichermassen heisst es für den geografischen Bildungsprozess, die Multiperspektivität von Weltbetrachtung herauszustellen. Nicht die Welt an sich wird durch AR ins Klassenzimmer geholt oder durch VR bereist, sondern immer nur eine bestimmte Betrachtungsweise von Welt, deren Autorenschaft es zu rekonstruieren gilt (vgl. Mohring und Brendel 2020).

Demgegenüber wird die $360^{\circ}$-Aufnahme auffällig oft als wertneutral beschrieben:

«Autorschaft scheint aus dem Bild ausradiert. Anstelle der Auswahl eines Kaders und der Kameraführung ist die gestalterische Freiheit des Kameramannes auf die Wahl der Kameraplatzierung limitiert» (Neußl 2018, 94).

Die zentralperspektivische Bildästhetik und panoptische Anordnung der $360^{\circ}$-Kameralinsen ("that look in every direction»; Milk 2015), die an die Ästhetik einer Überwachungskamera erinnern, legt jedoch ein Machtverhältnis zwischen Betrachtendem und Betrachtetem fest. Zudem ist der Aussenraum, in dem sich der Rezipient bzw. die Rezipientin während der VR-Erfahrung befindet, zumeist ein geschützter, körperlich nicht vulnerabler Raum. Eine Reflexion dieser Nicht-Übereinstimmung von Wahrnehmungssituation und wahrgenommener Situation (Seel 1998) sowie der im produktionsbezogenen Aussen manifestierten Machtkonstellation (Wer schaut an und wer wird angeschaut?) sollten berücksichtigt werden, wenn

14 Für eine kritische Auseinandersetzung mit Zeitzeugenschaft in geschichtswissenschaftlichen und -kulturellen Kontexten allgemein siehe (Welzer 2012; Sabrow 2012). 
VR als 〈empathy machine〉 betitelt und damit verbunden z. B. über Google Expeditions eine Klassenfahrt in ein jordanisches Flüchtlingslager angeboten wird (Nash 2018).

\section{Problemaufriss 2: Die unsichtbare Schnittstelle}

Auf thematischer Ebene werden für den Lehr- und Lernkontext Inhalte produziert, die mit dem Hineinversetzen in den oder dem Versetzen aus dem Bildrahmen eine Unmittelbarkeit der Lernerfahrung annehmen. Wie bereits dargelegt, ist auch dieses zugeschriebene Potenzial von AR und VR mit der Entrahmung der Bildlichkeit verknüpft: «Und man kann in alle Richtungen gucken. Man kann nach unten gucken, nach oben gucken und man erlebt Geschichte unmittelbar» («History 360 - das Making-of. Wie entstehen 360 Grad-Dokumentationen?» 2018). Anhand des Trailers für die im Herbst 2020 veröffentlichte Oculus Quest 2 wird im Folgenden die Annahme ebendieser Unmittelbarkeit in Bezug auf die Mensch-ComputerSchnittstelle, das sogenannte Interface, problematisiert.

Der Clip reiht VR an die Spitze einer Mediengeschichte ein, die als eine Geschichte der steten Steigerung einer sogenannten Naturalisierung der Bediengesten sowie Reduktion von materiellen und medialen Störungen erzählt wird. 

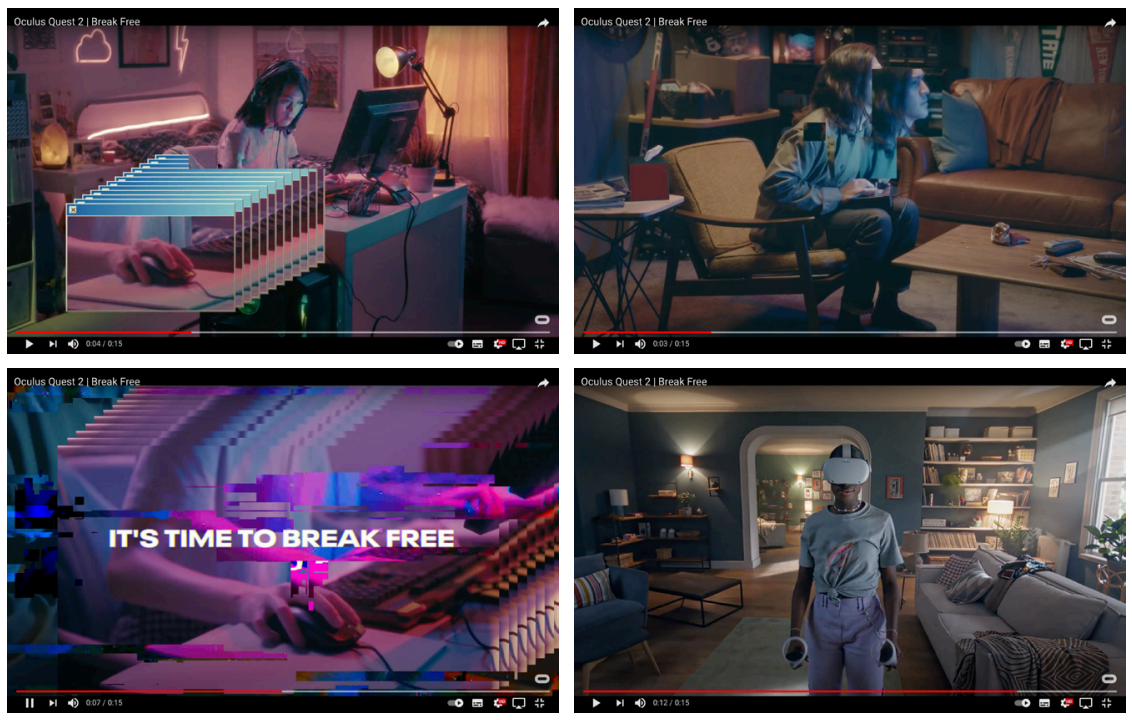

Abb. 2-5: Ausschnitte aus dem Trailer der Oculus Quest 2 (Oculus Quest 2 | Break Free 2020)

Die Bildsprache des Trailers sowie die sich wiederholende Liedzeile «My finger is on the button» inszenieren Knöpfe, Hebel, Maus und Tastatur als überholte und sperrige Mensch-Computer-Schnittstellen, die uunnatürliche> bzw. den Menschen an die Technik fesselnde Praktiken erforderten. Es sei also Zeit, sich von der einschränkenden und störanfälligen Technik zu befreien («It's time to break free») - mit der VR-Hardware Oculus Quest 2 stehe dieser wie auch immer gearteten freien Medieninteraktion nichts mehr im Wege.

Entgegen der im Trailer beworbenen Loslösung technischer Determiniertheit organisiert das Interface Bewegungs- und Wahrnehmungsformen jedoch mit, wie Schröter beispielsweise anhand des zentralperspektivischen Arrangements (Abb. 2-5) erläutert: 
«Ein perspektivisches Bild, welches sich mit der Kopfbewegung des Betrachters verändert [...], macht es dem Betrachter - im Unterschied zur Betrachtung statischer, perspektivischer Bilder - unmöglich, nicht den vorgesehenen Augenpunkt einzunehmen. Es gibt keine Möglichkeit, den von der apparativen Anordnung für das Subjekt vorgesehenen Platz gegenüber dem Bild zu verlassen [...].» (Schröter 2004, 192) $)^{15}$

Mit dem Bestreben, die Schnittstellen zwischen Mensch, Hard- und Software so unscheinbar und (natürlich〉(Natural User Interfaces) wie möglich zu gestalten, wird zum einen der Einfluss von materiellen und datentechnischen Komponenten auf Wahrnehmungsweisen und Wissensaneignungsprozesse ausgeklammert. Zum anderen bleibt unsichtbar, welche empirischen Befunde und welches, möglicherweise normierende Wissen über das Verhalten von Körpern in Form von Daten in die Anwendungen einfliessen. Um die Interaktion zwischen Mensch und Computer so störungsfrei wie möglich zu gestalten, spielt der Körper eine entscheidende Rolle, da er physisch und psychisch auf sensorische Reize reagiert und notwendige Bedingung für die Interaktion im und mit dem Virtuellen ist (Rieger 2018; Kaerlein 2018). Um eine Bewegung, zum Beispiel in einem SportSpiel, programmieren zu können, muss im Vorfeld Wissen über diese Körper, ihre Praktiken und Wahrnehmungsweisen explizit gemacht und in die Anwendung und Technik eingeschrieben werden. Wie Dawid Kasprowicz jedoch herausstellt, «dominiert das Bild einer Übertragung von Daten auf die menschlichen Sinneskanäle und zurück, wobei die Materialität dieser Kommunikation selten berücksichtigt wird» (Kasprowicz 2019, 20).

Im Rahmen des Sportunterrichts gilt es, bei der Nutzung von VRAngeboten dementsprechend zu reflektieren, welche Bewegungsformen vom medialen Apparat als richtig oder falsch identifiziert und somit im Bewegungsvollzug wirksam und eingeübt werden. Welche Subjektposition wird in den jeweiligen Anwendungen ermöglicht, welche verunmöglicht? In welche Rolle werden die Schülerinnen und Schüler also versetzt, als wer oder was werden sie von der virtuellen Umwelt adressiert und mit welchen

15 Kaerlein und Köhler sprechen diesbezüglich von einem «rregime of control> which, at first glance, contradicts the rhetorics of limitless freedom generally applied to VR» (Kaerlein und Köhler 2018, 185). 
Anforderungen und Verhaltensweisen sind diese Rollen verbunden? Welche Körpervorstellungen und -normen liegen den Anwendungen und der Annahme einer «natürlichen» Interaktion mit dem Interface zugrunde für welche Körper sind sie also ausgelegt?

\section{Wer, wen, was, wohin und wozu? Ein Fazit}

Die Potentiale, die AR und VR im Bildungskontext zugeschrieben werden, wurden von der Autorin unter einer Entrahmung der Bildlichkeit subsumiert, die ein Versetzen in andere Zeiten, Räume und Personen ermöglichen soll. Diese Eigenschaft wurde jedoch nicht als in den Technologien selbst angelegt oder fixiert betrachtet, sondern «nach ihren Voraussetzungen und der (strategischen) Funktion ihrer Existenz» (Stauff 2005, 166) gefragt. Dazu wurde das Zusammenspiel aus einer technologischen Neuerung in der Bildverarbeitung (virtueller Bildraum, der sich passend zur Blickrichtung und -perspektive entfaltet) und diskursiven Zuschreibungen (Unmittelbarkeit und Authentizität der Lernerfahrung, Freiheit des Blicks) rekonstruiert. Daran anschliessend hat der Beitrag die daraus resultierenden Implikationen bei der thematischen Ausgestaltung von AR- und VR-Angeboten für die Fächer Geschichte, Geografie und Sport problematisiert.

Obwohl die Wechselwirkungen aus Subjekten, Diskursen, Praktiken und Materialitäten, die die Medialität der beiden Technologien ausmacht (Weich 2020), nicht in Gänze in den Blick genommen werden konnten, kann der Titel «Wer versetzt wen oder was wohin - und wozu?» als Anregung für medienpädagogische und -didaktische Fragestellungen zu AR- und VR-Bildungsmedien dienen. Das Wer bezieht sich zum einen auf Grosskonzerne wie Facebook/Meta, die die Hardware für Augmented und Virtual Reality Technologie und Standards für die Betriebssoftware setzen. Zum anderen sind damit die entwickelnden Unternehmen der Programme und Anwendungen gemeint, zum Beispiel öffentlich-rechtliche Sendeanstalten oder auch nicht originär im Bildungssektor angesiedelte Unternehmen aus der Industrie oder Gamingbranche. Beide Seiten sind in je unterschiedliche polit-ökonomische Interessenskonstellationen eingebunden - ein Aspekt, der auch bei Verlagen von Schulbüchern oder anderen Distributoren von Bildungsmedien zu befragen ist, der in Anbetracht der grossen 
Datenmengen bei AR und VR jedoch eine neue Dimension erreicht. ${ }^{16}$ Hinzu könnten mit dem Wer auch jene Diskursakteurinnen und -akteure angesprochen werden, die in Auseinandersetzung mit AR und VR im Bereich Schule die Eigenschaften des Versetzens in den oder aus dem Bildrahmen fortschreiben. Das Wen fragt nach der Zielgruppe von Augmented und Virtual Reality Formaten. Das betrifft im Hinblick auf Schule nicht nur Alter, Klassenstufe oder Fach, sondern auch das vorausgesetzte Handhabungswissen - das auch immer eine medienkulturelle Dimension aufweist - und Fragen danach, für welche Körper eine VR- und AR-Anwendung eigentlich ausgelegt ist. Neben den physischen und psychischen Anforderungen an die Subjekte selbst könnte sich aus dem Wen die Frage ergeben, aus welcher Perspektive die Schülerinnen und Schülern die AR- oder VR-Anwendungen explorieren: Betreten sie die Arena als Profiboxerin/-boxer oder Amateursportlerin bzw. -sportler? Sind sie Beobachtende eines Geschehens oder werden sie als Teil der dargestellten Szenerie adressiert? Werden sie in der Rolle eines Guides oder Forschers mit einem digitalen Endgerät auf Erkundungstour geschickt? Mit Blick auf Augmented Reality fragt das Wen bzw. Was zudem nach den Personen oder Objekten, die - so der Anspruch der Produzierenden - in den "persönlichen Raum» (WDR 2019) versetzt werden sollen. Das Wohin kann wiederum Fragen adressieren, die sich mit dem Verhältnis von Virtualität und Realität beschäftigen, deren Hybridität bei AR und VR besonders virulent wird. Auch kann mit dem Wohin das vermeintliche Versetzen in andere Zeiten, Räume und Körper diskutiert werden. Das Wozu stellt die Frage nach der Kommunikationsabsicht: Zu welchem Zweck werden AR und VR im Bildungskontext eingesetzt? Welche Implikationen und Machteinschreibungen sind mit dem Versprechen an ein Versetzen in eine andere Zeit, einen anderen Raum oder Körper verknüpft und wie strukturieren diese die Rezeption von AR- und VR-Formaten mit? Auch lässt das Wozu die grundsätzliche ethische Diskussion zu,

16 Die Akteurinnen und Akteure liessen sich natürlich weiter ausdifferenzieren in z. B. produzierende Unternehmen einzelner technischer Komponenten wie Chips und Grafikkarten, Werbe- und PR-Agenturen oder auch Kanäle auf YouTube, die sich mit VR und AR auseinandersetzen. Aufgrund der Kürze des Artikels konnte hierauf leider nicht näher eingegangen werden. 
warum bestimmte Zeiten, Situationen und Perspektiven überhaupt nachgefühlt oder erlebt werden sollten und welcher Erkenntnisgewinn dadurch erhofft wird.

AR- und VR-Medien sind mehr als nur symbolische Gimmicks für die technische Aufrüstung des Klassenzimmers. Sie sind auch keine neutralen Tools oder Werkzeuge, sondern in ökonomische und bildungspolitische Konstellationen eingebunden, fungieren als Inklusions- und Exklusionsinstanzen, gestalten Nutzungs- und Wahrnehmungsweisen sowie die durch sie vermittelten Inhalte mit. Die oben genannten Problemaufrisse sind dabei keinesfalls spezifisch für AR- und VR-Technologien. Doch fehlt es jenseits bewahrpädagogischer Warnungen noch an kritischen Auseinandersetzungen mit AR und VR im Schulkontext, in denen die Materialität bzw. Medialität sowie wirtschaftliche Verschränkungen kritisch in den Blick genommen werden. Dabei ist zu beachten, dass die Präsentation oder Offenlegung eines produktionsbezogenen Aussen - der eingesetzten Technik, der Postproduktion etc. - oder der materiellen Schnittstellen nicht automatisch zu einer kritisch-reflexiven Haltung gegenüber selbigen Formaten führt (Bettinger, Draheim, und Weinrebe 2020). Eine didaktische Begleitung bleibt auch hier unabdingbar, um Medienbildungsprozesse nachhaltig anzustossen.

\section{Literatur}

Arnheim, Rudolf. 1932. Film als Kunst. Frankfurt a.M.: Suhrkamp.

Azuma, Ronald, Yohan Baillot, Reinhold Behringer, Steven K. Feiner, Simon Julier, und Blair MacIntyre. 2001. «Recent advances in augmented reality». IEEE Computer Graphics and Applications 21 (6): 34-47. https://doi.org/10.1109/38.963459.

Bettinger, Patrick, Saskia Draheim, und Paul Weinrebe. 2020. «Critical Making?: Praktiken in Makerspaces zwischen Widerständigkeit und Affirmation». Medienimpulse 58 (4): 1-34. https://doi.org/10.21243/mi-04-20-20.

Buchner, Josef. 2017. «Offener Geschichtsunterricht mit Augmented Reality». Medienimpulse 55 (1). https://journals.univie.ac.at/index.php/mp/article/view/ milo61.

Buehler, Kai, und Andreas Kohne. 2020. «Besser Lernen mit VR/AR Anwendungen». In Virtual Reality und Augmented Reality in der Digitalen Produktion, herausgegeben von Horst Orsolits und Maximilian Lackner, 75-97. Wiesbaden: Springer, Gabler. https://doi.org/10.1007/978-3-658-29009-2_5. 
Bunnenberg, Chirstian. 2018. «Virtual Time Travels? Public History and Virtual Reality». Public History Weekly 6 (3). https://doi.org/10.1515/phw-2018-10896.

Bürki, Rolf, und Josef Buchner. 2020. «Immersive Virtuelle Realität mit VR-Brillen im Geographieunterricht: Potentiale und Herausforderungen». Progress in Science Education 3 (2): 49-53. https://doi.org/10.25321/prise.2020.1001.

Curtis, Robin. 2016. «Immersion and Abstraction as Measures of Materiality». In Immersion in the Visual Arts and Media, herausgegeben von Fabienne Liptay und Burcu Dogramaci, 41-66. Leiden/Boston: Brill Rodopi.

Dreesen, Philipp, Łukasz Kumięga, und Constanze Spieß. 2012. «Diskurs und Dispositiv als Gegenstände interdisziplinärer Forschung. Zur Einführung in den Sammelband». In Mediendiskursanalyse. Diskurse - Dispositive - Medien Macht, herausgegeben von Philipp Dreesen, Łukasz Kumięga, und Constanze Spieß, 9-22. Wiesbaden: Springer. https://doi.org/10.1007/978-3-531-931487_1.

Facebook Connect I Keynote. 2020. https://www.youtube.com/watch?v=-cRxT32G7y4.

GfM, Gesellschaft für Medienwissenschaft. 2019. "Jahrestagung 2019: MedienMaterialitäten». GfM, Gesellschaft für Medienwissenschaft (blog). 17. Mai 2019. https://gfmedienwissenschaft.de/jahrestagung/archiv/2019-medien-materialitaeten.

Google. o. J. «Mit Expeditionen den Unterricht aufregend gestalten». Google for Education (blog). https://edu.google.com/intl/de_de/products/vr-ar/expeditions/.

Grau, Oliver. 2001. Virtuelle Kunst in Geschichte und Gegenwart. Visuelle Strategien. Berlin, Bonn: Dietrich Reimer.

Henze, Niels, Jochen Koubek, Katrin Wolf, Moritz Puppel, Jens Reinhardt, und Rufat Rzayev. 2019. «GEVAKUB: Gestaltungsrichtlinien für virtuelle Lernräume in der kulturellen Bildung». In Forschung zur Digitalisierung in der Kulturellen Bildung, herausgegeben von Benjamin Jörissen, Stephan Kröner, und Lisa Unterberg, 93-108. München: kopaed.

Hicketier, Knut. 1999. «Fenster zum Welt und Weltersatz - das Fernsehen». In Auf dem Weg zur 'Weltkultur', herausgegeben von Brockhaus, 6: 220-28. Kunst und Kultur. Leipzig, Mannheim: Brockhaus.

«History $360^{\circ}$ - das Making-of. Wie entstehen 360 Grad-Dokumentationen?» 2018. Making-of. https://www.zdf.de/geschichte/history-360-grad/history360-making-of-100.html.

Jauch, Steffen. 2019. «Virtual und Augmented Reality im Klassenraum? Ein Überblick bildungsrelevanter Angebote». bpb (blog). 2019. https://www.bpb.de/lernen/digitale-bildung/werkstatt/298516/virtual-und-augmented-reality-imklassenraum-ein-ueberblick-bildungsrelevanter-angebote.

Jordan, Stefan. 2013. Theorien und Methoden der Geschichtswissenschaft. 2. Aufl. Paderborn: Schöningh. 
Kaerlein, Timo. 2018. Smartphones als digitale Nahkörpertechnologien. Zur KYbernetisierung des Alltags. Paderborn: transcript. https://doi. org/10.1515/9783839442722.

Kaerlein, Timo, und Christian Köhler. 2018. «Around a Table, around the World. Facebook Spaces, Hybrid Image Space and Virtual Surrealism». In Image - Action - Space. Situating the Screen in Visual Practice, herausgegeben von Luisa Feiersinger, Kathrin Friedrich, und Moritz Queisner, 177-90. Berlin, Boston: De Gruyter. https://doi.org/10.25969/mediarep/12208.

Kasprowicz, Dawid. 2019. Der Körper auf Tauchstation: Zu einer Wissensgeschichte der Immersion. Baden-Baden: Nomos. https://doi.org/10.5771/9783845298689-1.

kmz. 2020. «Augmented Reality: Apps und Dienste». Kreis-Medienzentrum Ludwigsburg (blog). 2020. https://kmz-ludwigsburg.de/augmented-reality-appsund-dienste-welche-augmented-reality-apps-und-dienste-gibt-es-im-bildungsbereich-ein-ueberblick/.

Krech, Hubert. 2019. «Virtual Reality im Unterricht - Über dir der Rosinenbomber». ZDF (blog). 2019. https://www.zdf.de/nachrichten/heute/auf-zeitreiseschueler-testen-zdf-history36o-grad-10o.html?sfns=mo.

Lenzen, Kirstin. 2020. Die multiple Identität der Technik Eine Innovationsbiographie der Augmented Reality-Technologie. Bielefeld: transcript. https://doi. org/10.14361/9783839451854.

Liu, Dejian, Chris Dede, Ronghuai Huang, und John Richards, Hrsg. 2017. Virtual, Augmented and Mixed Realities in Education. Singapur: Springer. https://doi. org/10.1007/978-981-10-5490-7.

Maas, Melanie J., und Janette M. Hughes. 2020. «Virtual, augmented and mixed reality in K-12 education: a review of the literature». TechnologY, Pedagogy and Education 29 (2): 231-49. https://doi.org/10.1080/1475939X.2020.1737210.

Making of WDR History App. o. J. Making-of. https://wwwl.wdr.de/mediathek/video/sendungen/video-making-of-wdr-history-app-10o.html.

Medienzentrum Pforzheim-Enzkreis. o. J. «Medienzentrum Pforzheim-Enzkreis». https://padlet.com/MZPE.

Milgram, Paul, und Fumio Kishino. 1994. "A Taxonomy of Mixed Reality Visual Displays». IEICE Transactions on Information Systems E77-D (12): 1321-29.

Milk, Chris. 2015. «How Virtual Reality Can Create the Ultimate Empathy Machine». TED-Talk. https://www.ted.com/talks/chris_milk_how_virtual_reality_can_create_the_ultimate_empathy_machine?language=de.

Mittelstaedt, Tim. 2021. «timmersive». timmersive (blog). 2021. https://timmersive.eu.

Mohring, Katharina, und Nina Brendel. 2020. «Virtual-Reality-Exkursionen im Geographiestudium - neue Blicke auf Virtualität und Raum». In Augmentierte und virtuelle Wirklichkeiten, herausgegeben von Andreas Beinsteiner, Lisa Blasch, Theo Hug, Petra Missomelius, und Michaela Rizzolli, 189-204. Innsbruck: Innsbruck University Press. https://library.oapen.org/handle/20.500.12657/46239. 
Mulders, Miriam, und Josef Buchner. 2020. "Lernen in immersiven virtuellen Welten aus der Perspektive der Mediendidaktik». Medienimpulse 58 (2): 1-23. https://doi.org/10.21243/mi-02-20-22.

Nash, Kate. 2018. «Virtual reality witness: exploring the ethics of mediated presence». Studies in Documentary Film 12 (2): 119-31. https://doi.org/10.1080/1750 3280.2017.1340796.

Neußl, Patrick. 2018. «360 Video - Immersion als mediales Dispositiv». Masterarbeit, Universität Wien. https://doi.org/10.25365/thesis.54251.

nextReality. Hamburg. 2021. Meetup / Potenziale von VR im Sport - Gute Vorsätze mit VR in die Realität umsetzen. Meetup. https://www.youtube.com/ watch?v=K93WpXlEwAo.

Oculus Quest 2 |BreakFree.2020. https://www.youtube.com/watch?v=l2bo2sUKfoI.

Pölert, Hauke. 2019. "Augmented Reality (AR) im Geschichtsunterricht - 5 Praxisbeispiele». unterrichten. digital (blog). 2019. https://unterrichten.digital/2019/12/og/augmented-reality-geschichtsunterricht/.

Radu, Iulian. 2014. "Augmented reality in education: a meta-review and crossmedia analysis». Personal and Ubuquitous Computing 18: 1533-43. https://doi. org/10.1007/s00779-013-0747-y.

Rieger, Stefan. 2018. " "Bin doch keine Maschine..." Zur Kulturgeschichte eines Topos». In Machine Learning - Medien, Infrastrukturen und Technologien der Künstlichen Intelligenz, herausgegeben von Christoph Engemann und Andrea Sudmann, 117-42. Bielefeld: transcript. https://doi.org/10.1515/9783839435304006.

Sabrow, Martin. 2012. «Der Zeitzeuge als Wanderer zwischen zwei Welten». In Die Geburt des Zeitzeugen nach 1945, herausgegeben von Martin Sabrow und Norbert Frei, 1. Aufl., 414: 13-32. Geschichte der Gegenwart. Göttingen: Wallstein.

Schröter, Jens. 2004. Das Netz und die Virtuelle Realität. Zur Selbstprogrammierung der Gesellschaft durch die universelle Maschine. Bielefeld: transcript. https://doi. org/10.25969/mediarep/3737.

Seel, Martin. 1998. «Medien der Realität und Realität der Medien». In Medien, Computer, Realität. Wirklichkeitsvorstellungen und Neue Medien, herausgegeben von Sybille Krämer. Frankfurt am Main: Suhrkamp. gekürzte Fassung verfügbar unter http://www.medien-gesellschaft.de/html/seel-_medienrealitat.html.

Stauff, Markus. 2005. „Das neue Fernsehen'. Machtanalyse, Gouvernementalität und digitale Medien. Medien'Welten. Braunschweiger Schriften zur Medienkultur 2. Münster: LIT. https://doi.org/10.25969/mediarep/11ll.

Stern, Martin. 2019. «Körper - Medien - Selbst in neuen Sportpraktiken». In SelfTracking, Selfies, Tinder und Co. Konstellationen von Körper, Medien und Selbst in der Ge-genwart, herausgegeben von Daniel Rode und Martin Stern, 37-54. Bielefeld: transcript. https://doi.org/10.1515/9783839439081-002.

Stiftung Lesen, Hrsg. 2018. Virtual Reality. Virtuelle Welten entdecken, Wissen erleben. Mainz: Stiftung Lesen. https://www.derlehrerclub.de/download. php?type=documentpdf\&id=2090. 
Stiftung Lesen, Hrsg. 2019. Augmented Reality im Unterricht. Neue Perspektiven für das Lernen und Lesen. Mainz: Stiftung Lesen. https://www.derlehrerclub.de/ download.php?type=documentpdf\&id=2091.

Sutherland, Ivan E. 1968. «A head-mounted three dimensional display». Fall Joint Computer Conference, 757-64.

Time \& History. o. J. «Führerbunker VR. Eine interaktive Dokumentation über die letzten Tage des Dritten Reichs im 〈Führerbunker〉». Homepage. Time \& History (blog). https://timeandhistory.com/de/home_ger/.

«Virtual Reality - Schöne neue Welt?». 2020. phoenix plus. https://www.phoenix. de/sendungen/ereignisse/phoenix-plus/virtual-reality---schoene-neue-welta-1378580.html.

Wagner, Jens-Christian. 2019. «Simulierte Authentizität? Chancen und Risiken von Augmented und Virtual Reality an Gedenkstätten». Gedenkstättenrundbrief 196 (Dezember): 3-9.

WDR. 2019. «Erfolge: Grimme, Prix Europa \& Co.». 2019. https://www1.wdr.de/ fernsehen/unterwegs-im-westen/ar-app/ar-app-info-10o.html.

Weich, Andreas. 2020. «Hervorbringung von Medienkonstellationen statt Nutzung didaktischer Werkzeuge. Versuch einer medienkulturwissenschaftlichen Didaktik der Bildungsmedien am Beispiel von Videokonferenzen als Unterrichtsform». Medienimpulse 58 (2): 1-32. https://doi.org/10.21243/mi-0220-20.

Welzer, Harald. 2012. «Vom Zeit- zum Zukunftszeugen. Vorschläge zur Modernisierung der Erinnerungskultur». In Die Geburt des Zeitzeugen nach 1945, herausgegeben von Martin Sabrow und Norbert Frei, 1. Aufl., 414:33-48. Geschichte der Gegenwart. Göttingen: Wallstein.

Wössner, Stephanie. o. J. «Virtual Reality: Apps und Dienste». Landesmedienzentrum Baden-Württemberg (blog). https://www.lmz-bw.de/medien-und-bildung/ medienwissen/virtual-und-augmented-reality/virtual-reality-apps-unddienste/\#/medien-und-bildung/medienwissen/virtual-und-augmented-reality/virtual-reality-apps-und-dienste/.

ZDF. o. J. «Geschichte hautnah erleben». ZDF-History 360 (blog). https://www.zdf. de/geschichte/history-360-grad\#xtor=CS5-4.

Zobel, Benedikt, Sebastian Werning, Dirk Metzger, und Oliver Thomas. 2018. «Augmented und Virtual Reality: Stand der Technik, Nutzenpotenziale und Einsatzgebiete». In Handbuch Mobile Learning, herausgegeben von Claudia de Witt und Christina Gloerfeld, 123-40. Wiesbaden: Springer. https://doi. org/10.1007/978-3-658-19123-8_7. 